\title{
Investigating the Super Paramagnetic Limits of Ferromagnetic Nanowires
}

\author{
C. V. Ezeh ${ }^{1 *}$, J. C. Ehiabhili ${ }^{2}$ and D. A. Allwood ${ }^{1}$ \\ ${ }^{1}$ Sheffield Centre for Advanced Magnetic Materials and Devices, United Kingdom \\ ${ }^{2}$ Department of Physics, Federal University Oye-Ekiti, Ekiti State, Nigeria \\ *Corresponding author email: chioma.odoh [AT] fuoye.edu.ng
}

\begin{abstract}
Highly sensitive focused magneto optical Kerr effect magnetometer (MOKE) has been used to explore the nanoscale limit of state of the art electron beam lithography (EBL) patterned permalloy (NiFe) nanowires. The nanowires are $20 \mu \mathrm{m}$ long arranged in $20 \mu \mathrm{m} \times 20 \mu \mathrm{m}$ arrays. Permalloy of different thicknesses, which includes 10, 8 , 6,4 and $3.5 \mathrm{~nm}$ were deposited on 5 different samples containing these arrays. Results from $10 \mathrm{~nm}$ thick permalloy nanowires shows superparamagnetic transition at $85 \pm 5 \mathrm{~nm}$ wire width. For 6 nm thick array, superparamagnetic transition occurs at $125 \pm 25 \mathrm{~nm}$. We therefore show that with $10 \mathrm{~nm}$ thickness, instead of $6 \mathrm{~nm}$ thickness, more miniaturisation of nanowire width can be achieved before the onset of superparamagnetism. This may be seen as very efficient in achieving increased storage density in memory devices.
\end{abstract}

Keywords--- Permalloy, nanowires, coercivity, superparamagnetic transition

\section{INTRODUCTION}

The inherent property of ferromagnets to align their magnetic moment strongly in the direction of applied magnetic field is threatened as the magnets are miniaturized down to sub hundreds and tenths of nanometer. Apart from their strong magnetization in the non miniaturized state, they retain internally induced magnetic field even when the previously induced external field is removed [1]. Paramagnets on the opposite has randomly arranged and weakly coupled magnetic moments and do not retain their internal induced magnetic field once the applied field is removed [2]. Superparamagnetism occurs in further miniaturized ferromagnets [3] - a phenomenon whereby ferromagnetic materials respond to applied external magnetic field in a similar way to that of a paramagnet.

Ferromagnetic nanowires have received more attention due to their applications in magnetic logic devices and racetrack memory. They are also used to achieve increased storage density in memory devices and long term stability of memory or recording. As the width of ferromagnetic nanowires decreases, intensity of the magnetic field needed to reduce their magnetisation to zero after saturation magnetisation has been achieved (coercivity) increases rapidly. In other words, ferromagnetic nanowire experiences an inverse width relationship as show in equation 1 with this magnetization reversal from film thicknesses $\geq 3.5 \mathrm{~nm}$ [3]. This shows the tremendous effect of geometry of a magnetic sample on the magnetization reversal.

$$
H_{c}=a+\frac{\left(3 M_{S} d\right)}{W}
$$

where $\mathrm{W}$ is the width of the nanowire, $\mathrm{M}_{\mathrm{s}}$ is saturation magnetization, $\mathrm{H}_{\mathrm{c}}$ is coercive field and $\mathrm{d}$ is thickness of the magnetic material deposited on the wire. The inverse width dependence occurs because magnetization reversal is controlled by a small nucleus that spreads across the width of a nanowire but does not depend on the length of the wire. However, with further reduction in the width and thickness of a nanowire, size of the controlling nucleus approaches superparamagnetic limit, which is a region of sudden decrease in coercivity with decreasing wire width. As a result, the inverse width relationship with magnetization reversal breaks down. A curve equivalent to that shown in Figure 1 is an example of superparamagnetism (a sudden decrease in coercivity with decreasing wire width) for $2.5 \mathrm{~nm} \mathrm{NiFe}$ lines [3]. 


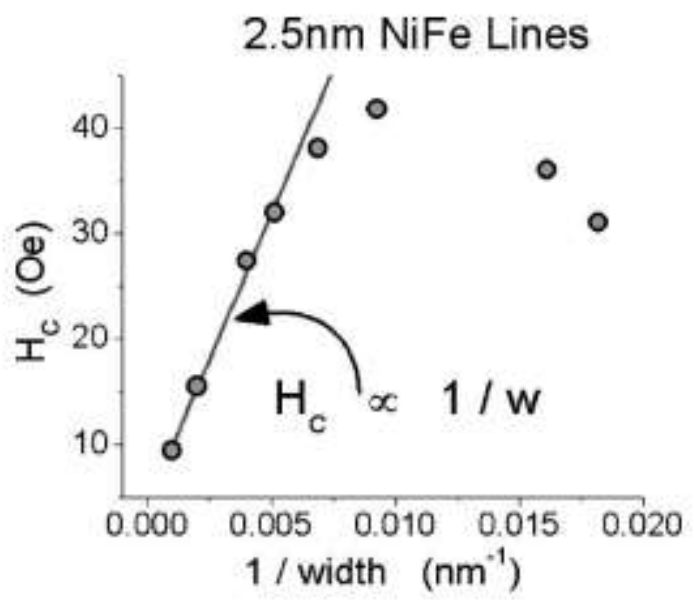

Figure 1: Trend showing sudden decrease in coercivity with decreasing wire width for a very thin wire of $2.5 \mathrm{~nm}$ superparamagnetic transition [3].

\section{EXPERIMENT}

State of the art electron beam lithography (EBL) can be used to make nanowire patterns with diameter down to tens of nm. In this research, room temperature EBL patterned $20 \mu \mathrm{m} \times 20 \mu \mathrm{m}$ arrays of $20 \mu \mathrm{m}$ long Permalloy (NiFe) nanowires has been fabricated. The width of the nanwires ranges between 10 to $500 \mathrm{~nm}$, which includes 10, 20, 30, 40, 50, 60, 70, 80, 90, $100,150,200,300,400$ and $500 \mathrm{~nm}$ with varying thickness of 3.5 to $10 \mathrm{~nm}$ and wires of the same width arranged in the same array. Nanowires in an array are separated by twice their width while $50 \mu \mathrm{m}$ gaps separate one array from the other.

The whole process fabrication of the NiFe nanowires involved nanowire design with LibreCAD, patterning with electron beam lithography (EBL), deposition of permalloy with thermal evaporator, measurement with magneto optical Kerr effect (MOKE) magnetometer and imaging and section analysis with atomic force microscope (AFM). A concise and comprehensive list of the materials and equipment used for each stage of the research is given in Table 1 below.

\begin{tabular}{|c|c|c|c|}
\hline $\mathbf{S} / \mathbf{N}$ & ACTIVITY & $\begin{array}{l}\text { METHOD/ } \\
\text { EQUIPMENT USED }\end{array}$ & CHEMICAL USED \\
\hline 1 & Design of nanowires & $\begin{array}{l}\text { Computer aided design- } \\
\text { LibreCAD/ Snap to the grid } \\
\text { command. }\end{array}$ & Not applicable \\
\hline 2 & $\begin{array}{l}\text { Patterning of the } \\
\text { nanowires }\end{array}$ & $\begin{array}{l}\text { Electron beam lithography } \\
\text { (EBL), silicon substrate, spin } \\
\text { coater, hot plate for soft baking. }\end{array}$ & $\begin{array}{l}\text { Acetone for cleaning of silicon substrate, isopropyl } \\
\text { alcohol (IPA) for washing off of acetone, } \\
\text { Polymethylmethacrylate (PMMA) as the positive } \\
\text { resist, silicon substrate, }\end{array}$ \\
\hline 3 & $\begin{array}{l}\text { Deposition of permalloy } \\
(\mathrm{NiFe})\end{array}$ & Thermal evaporator & $\begin{array}{l}\text { Polymethylmethacrylate (PMMA) as a mask and } \\
\text { for sticking the silicon substrate onto the sample } \\
\text { holder, NiFe as the magnetic material deposited on } \\
\text { the patterned silicon substrate, acetone for lift-off, } \\
\text { isopropyl alcohol (IPA) for rinsing of the acetone, } \\
\text { hand gloves and tweezers. }\end{array}$ \\
\hline 4 & $\begin{array}{l}\text { Measurement of } \\
\text { magnetic properties }\end{array}$ & $\begin{array}{l}\text { Focused magneto optical Kerr } \\
\text { effect (Focused MOKE) } \\
\text { magnetometer }\end{array}$ & Not applicable \\
\hline 5 & $\begin{array}{l}\text { Imaging and section } \\
\text { analysis }\end{array}$ & $\begin{array}{l}\text { Atomic force microscope } \\
(\mathrm{AFM}) / \text { contact mode probe }\end{array}$ & Not applicable \\
\hline
\end{tabular}

Table 1: List of equipment and materials used in the research

\section{Design of the Magnetic Nanowire Structures}

LibreCAD is 2D and geometry computer aided design (CAD) which is able to file in the industrially accepted GDSII format. Snap to grid command contained in it makes it easy for basic shapes to be drawn with high dimension accuracy. With LibreCAD, $20 \mu \mathrm{m} \times 20 \mu \mathrm{m}$ arrays of $20 \mu \mathrm{m}$ long wires were designed. The wire width ranges from 10 to $500 \mathrm{~nm}$ and includes 10, 20, 30, 40, 50, 60, 70, 80, 90, 100, 150, 200, 300, 400 and $500 \mathrm{~nm}$ with wires of the same width arranged in an array and separated by twice their width. $50 \mu \mathrm{m}$ gaps separate one array from the other. Different shapes of marker 
pad were designed $300 \mu \mathrm{m}$ away above each array for easy identification of the nanowire arrays during measurement. Figure 2 shows the schematic diagram of the arrangement of the nanowire structures.

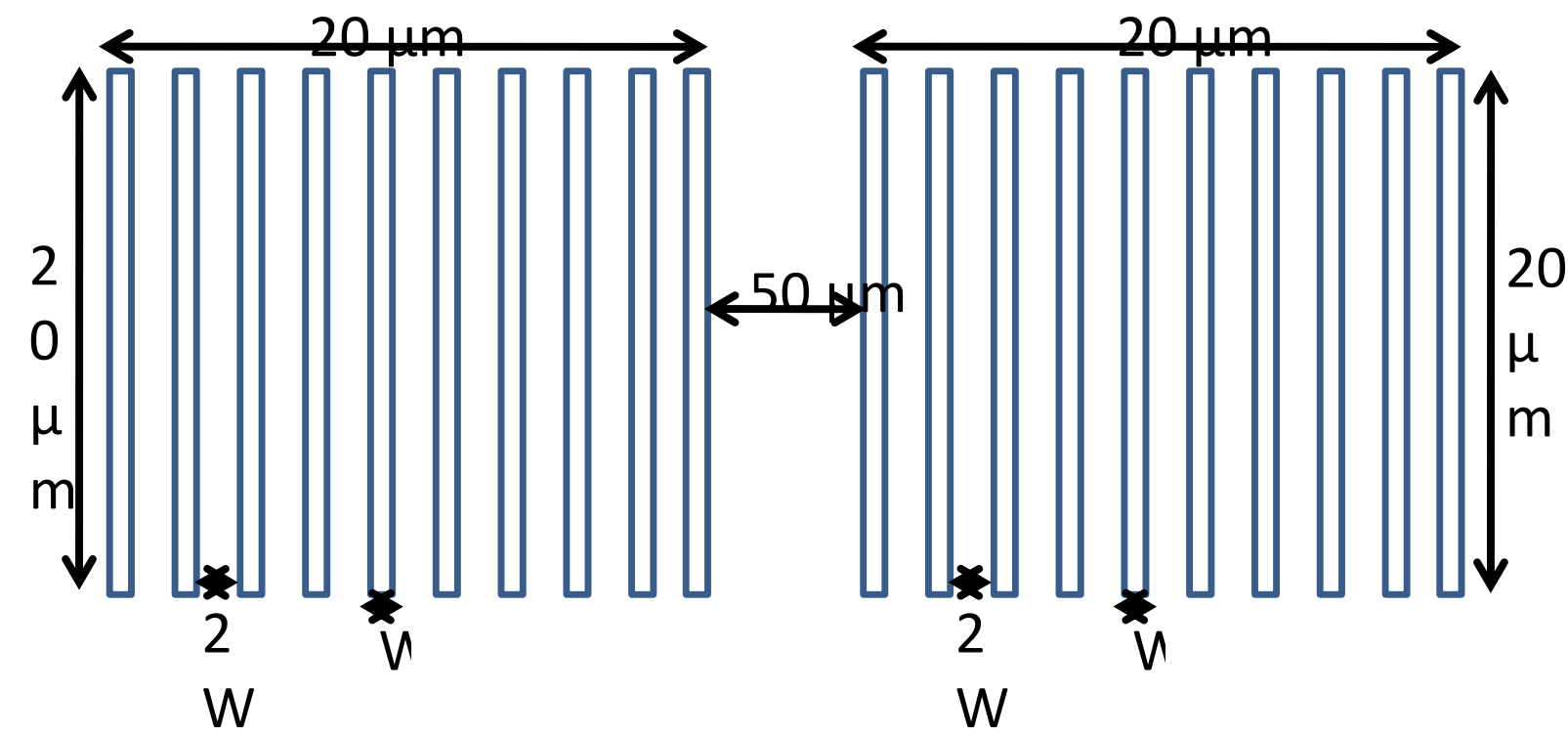

Figure 2: Schematic diagram of (a) $20 \mu \mathrm{m} \times 20 \mu \mathrm{m}$ arrays of $20 \mu \mathrm{m}$ long. $* \mathrm{~W}$ represents different nanowire width, which includes 10, 20, 30, 40, 50, 60, 70, 80, 90, 100, 150, 200, 300, 400, and $500 \mathrm{~nm}$. nanowires of the same with are arranged in the same array.

\section{Patterning the Nanowires}

EBL patterning of the nanostructures were performed with Joel JBX 6300FS electron beam lithography system at the University of Leeds, United Kingdom. Its minimum feature size is $<8 \mathrm{~nm}$ with beam-electron overlay accuracy of $<25$ $\mathrm{nm}$. The accelerating voltage is $100 \mathrm{KV}$ and beam currents from $30 \mathrm{pA}$ to $20 \mathrm{nA}$. Due to nano dimensions of the designed structures, 5 atomically flat $\mathrm{SiO}_{2}$ substrates unto which the patterns were transferred were first cleaned in $50^{\circ} \mathrm{C}$ acetone for 5 minutes, after which it was rinsed in cold acetone then isopropyl alcohol (IPA) and dried with gaseous nitrogen. $2 \%$ solution of PMMA in anisole was spin coated on the substrates at $1000 \mathrm{rpm}$ for $10 \mathrm{~s}$. Then the substrates were soft baked at $50^{\circ} \mathrm{C}$ for 1 hour. Afterwards, the substrates were transferred into the vacuum chamber of the EBL system and exposed to the electron beam following the computer generated pattern. The exposed substrates were developed by immersion in methyl-isobutyl-ketone (MIBK) in the ratio 1:3 with IPA. Afterwards, they went through ultra sound agitation for 30 seconds.

\section{Permalloy Deposition}

Thermal evaporation and sputtering are methods that can be used to deposit magnetic materials unto a substrate. With thermal evaporation, very thin films (mostly metals) can be deposited onto a substrate in a high vacuum environment of about $10^{-7}$ mbar, which allows vapour particles from the target material to travel directly to the substrate. It deposits in the thickness range of angstroms to microns and requires low voltage and high current to function. In sputtering, atoms are removed from a target by impact from ions of inert gas plasma like Argon. A high vacuum chamber is filled with the inert gas and voltage applied across the gas creates plasma. Positive ions generated accelerates to the cathode where material to be deposited is placed and sputters atoms from it, which condenses unto the substrate.

In this research, thermal evaporator was used to deposit permalloy (NiFe) onto 5 different EBL patterned substrates- 1,2,3,4 and 5. Each substrate differs from the other only by the thickness of permalloy deposited on them. Substrates 1,2,3,4 and 5 contains $10,8,6,4$ and $.5 \mathrm{~nm}$ thick NiFe respectively. The thermal evaporator uses tungsten basket. The base pressure of the vacuum chamber was $3 \times 10^{-7} \mathrm{mbar}$, which increased to $7 \times 10^{-6} \mathrm{mbar}$ during evaporation. Lift-off was done for 60 seconds in acetone by ultrasonic vibration and the samples were rinsed in IPA.

\section{Magneto Optical Kerr Effect Magnetometer}

Magneto optical Kerr effects in magnetic materials arise due to the optical anisotropy of such materials. These materials have magnetisation within surface domains, which can be influenced by magnetic field [4]. MOKE is similar to Michael Faraday effect where the polarisation of light is rotated through a transparent material subjected to a magnetic field. The operational principle is that when polarised light is incident on a magnetic material, it reflects and changes its polarisation. Change in polarisation is proportional to magnetisation. Therefore, through measuring change in polarisation, magnetisation of the magnetic material of interest can be deduced. 
MOKE is a non-destructive technique for measuring magnetization in magnetic materials. Due to its high spatial resolution, which is still developing, and high sensitivity, it remains at the forefront of magnetic nanostructure research. With it, domain wall propagation in ultrathin magnetic nanowires has been imaged [5] and the magnetisation response of the individual structures studied on ultra-fast time scale with a spatial resolution <200 $\mathrm{nm}$ [6]. Also, it has been used to study isolated magnetic microstructures $[7,8]$, behaviour of nanodot chains $[9,10]$ and even the behaviour of selected regions of separate nanowire structures $[11,12]$.

For sample imaging, white light that is brought collinear with the laser optical axis by means beam splitter between the objective lens and polariser forms the imaging optical path. Dichroic mirror placed before the CCD chip filters intense laser beam. While the sample surface is imaged unto a CCD camera through the beam splitter placed just before the $\lambda / 4$ wave plate. Iterative rotation of the analyser first then $\lambda / 4$ wave until a minimum voltage is achieved, followed by rotation of the analyser by $20^{\circ}$ for further voltage reduction sets up the polarization optics for measurement. Diode-pumped solid state laser (coherent Verdi) with operating wavelength of $532 \mathrm{~nm}$ and continuously variable output power $\sim 5 \mathrm{~W}$ kicks off the measurement procedure. The ring cavity has excellent low stability characteristic of about $0.02 \%$, measured between $10 \mathrm{~Hz}$ to $1 \mathrm{GHz}$. Therefore, high power output, half- wave plate /polariser system and neutral density (ND) filter are used in attenuating the laser to obtain stability characteristic close to that of the laser light. The beam polarisation ratio is improved by allowing it pass through beam expander and a high quality Glan-Taylor polarizing prism with extinction ratio of $<10^{-5}$. The beam focuses on $\sim 5 \mu \mathrm{m}$ diameter spot of the sample. Reflection from the spot is collected with identical chromate doublet lenses with infinite conjugate ratios and $500 \mathrm{~mm}$ focal length. The focused laser is supposed to have perfect or at least near perfect Gaussian profile in the plane of incidence but does not because the sample is mounted at $45^{\circ}$ to the optical axis. This is achieved by having the objective lens adjusted until the focused spot has high central intensity that is sufficient without significant higher order mode being noticed. The laser beam is then directed to a Glan-Taylor polarising analyser through a $\lambda / 4$ wave guide retardation plate, which introduces a relative phase shift of $90^{\circ}$ in the reflected light. Finally, Silicon photodiode detects the reflected beam, providing Kerr signal.

Photodiodes are connected to the lock in amplifier, which features $\pm 2 \mathrm{~V}$ offset adjustment. The offset is adjusted as the detectors are illuminated until each amplifier gives a net output signal close to zero, though the offset is later added to the signal as part of data acquisition. This improves the effective dynamic range of the measurement. Data averaging occurs in the digital storage oscilloscope before being downloaded to the PC for analysis. Magnetic field is generated in the plane of the sample by electromagnets and iron-core driven by KEPCO power amplifier [13]. TTI function generator defines the waveforms. XY $\theta$ stage is a computer controlled translation stage with 55nm lateral resolution where magnetic samples are mounted. CCD camera image guides in placing the sample well. The sample is raster scanned through a two-dimensional grid beneath the laser spot, which is recorded with the signal from the lock-in amplifier for each position in the grid. The signal allows susceptibility map of the sample to be generated. Using this, a specific area in the magnetic structure can be studied as well as individual magnetic nanostructures identified $[14,15]$.

\section{RESULTS}

To check for superparamagnetic transition (SPT) in the nanowires, plots of inverse of the designed nanowire widths versus coercivity $\mathrm{H}_{\mathrm{c}}$ for samples 1 (10 nm thick) and 3 (6 nm thick) wires were made as shown in Figure 3 . Following the trend of inverse width relationship with coercivity discussed earlier, SPT is evident in the plots. $10 \mathrm{~nm}$ thick nanowires in Figure 3 (a) shows SPT between 0.0111 and $0.0125 \mathrm{~nm}^{-1}$. This gives the wire width of about $85 \pm 5 \mathrm{~nm}$. The SPT of $85 \pm 5 \mathrm{~nm}$ can also be clearly seen in the graph of $\mathrm{H}_{\mathrm{c}}(\mathrm{Oe})$ versus width $(\mathrm{nm})$ shown in figure $3(\mathrm{~b})$. Result from 6 nm thick nanowires is shown in figure 4. Figure 4 (a) shows SPT between 0.007 and $0.01 \mathrm{~nm}^{-1}$, which is about $125 \pm 25 \mathrm{~nm}$. The SPT of 125 $\pm 25 \mathrm{~nm}$ can also be seen clearly in the graph of $\mathrm{H}_{\mathrm{c}}(\mathrm{Oe})$ versus width (nm) shown in figure 4 (b). 
a

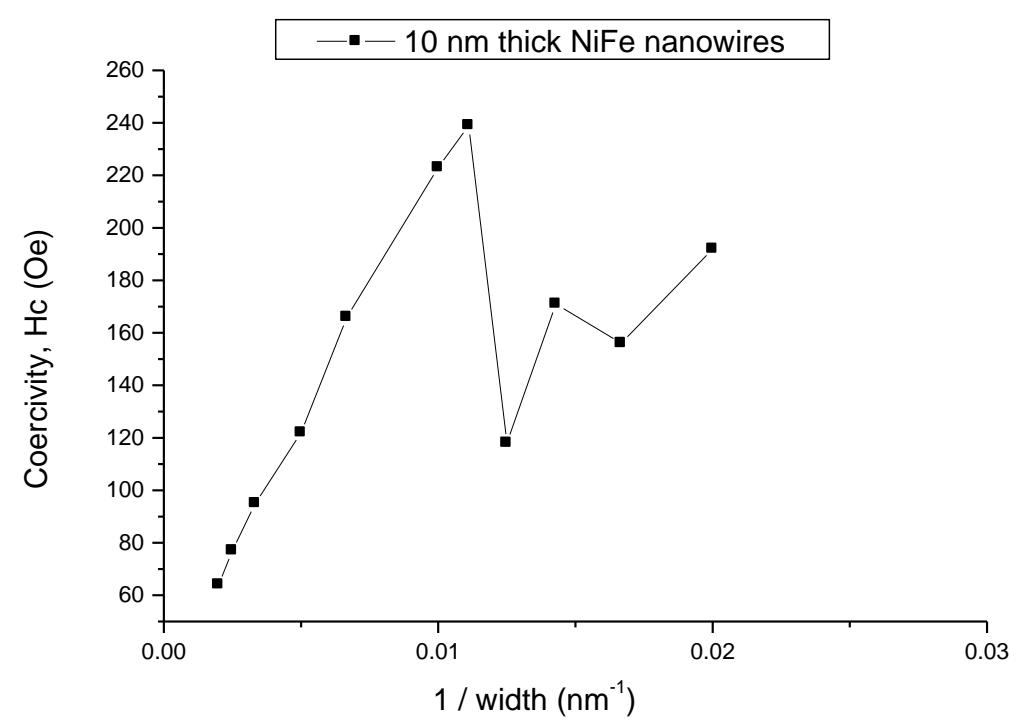

b

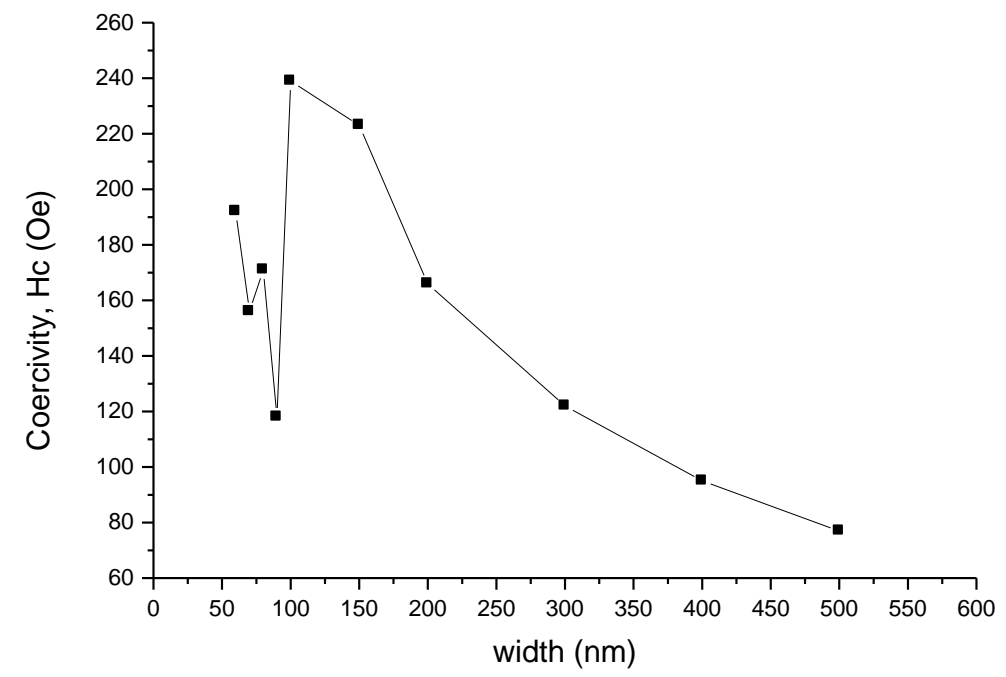

Figure 3: Result from sample 1 (10 nm thick nanowires). (a): Plot of coercivity, $\mathrm{H}_{\mathrm{c}}(\mathrm{Oe})$ versus inverse of nanowire width showing superparamagnetic transition between 0.0111 and $0.0125 \mathrm{~nm}^{-1}$ (b) Plot of coercivity, $\mathrm{H}_{\mathrm{c}}(\mathrm{Oe})$ versus nanowire width showing SPT at $85 \pm 5 \mathrm{~nm}$. 
a

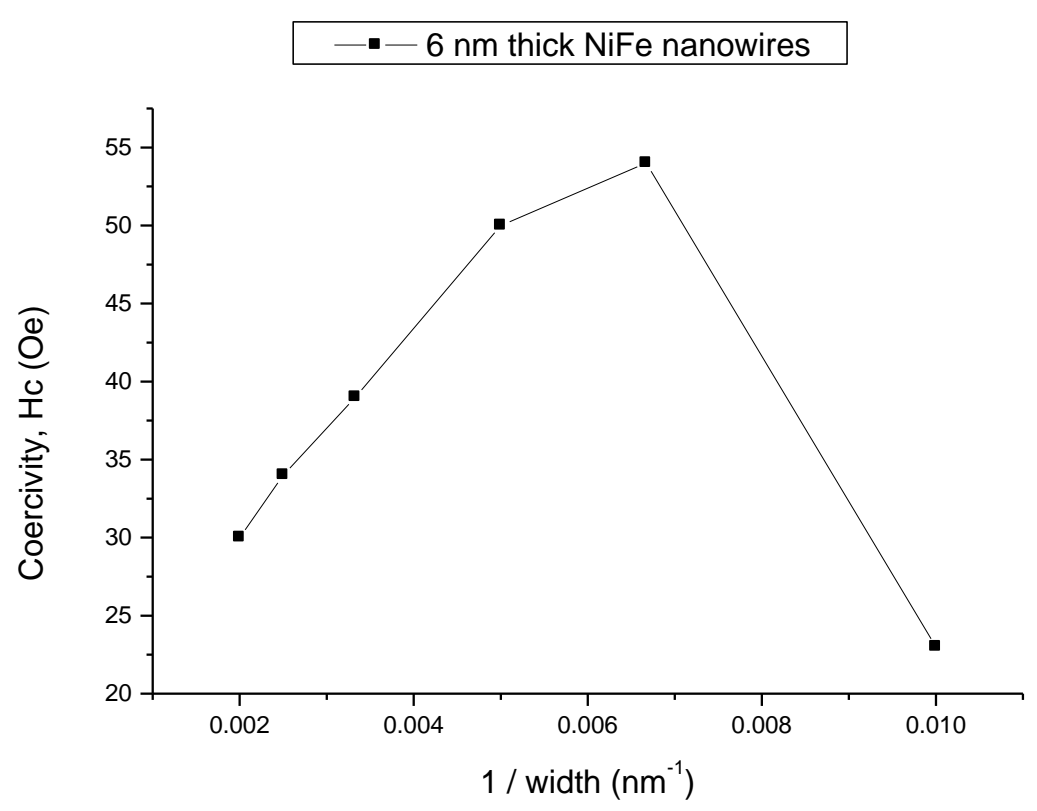

b

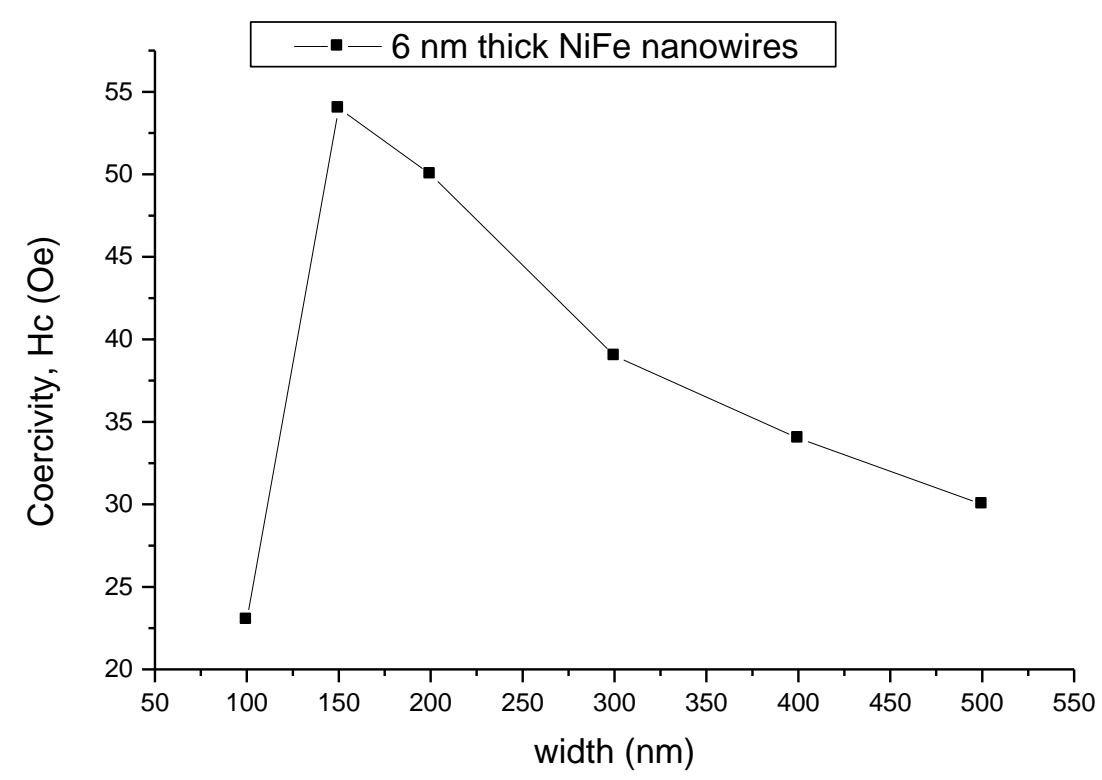

Figure 4: Result from sample 2 (6 nm thick nanowires). (a): Plot of coercivity, $\mathrm{H}_{\mathrm{c}}(\mathrm{Oe})$ versus inverse of nanowire width showing superparamagnetic transition between 0.007 and $0.01 \mathrm{~nm}^{-1}$ (b) Plot of coercivity, $\mathrm{H}_{\mathrm{c}}(\mathrm{Oe})$ versus nanowire width showing SPT at $125 \pm 25 \mathrm{~nm}$.

\section{CONCLUSION}

We have investigated superparamagnetic transition in permalloy nanowires made by Electron Beam Lithography and Thermal Evaporation. We show that superparamagnetism sets in at wire width of $85 \pm 5 \mathrm{~nm}$ and $10 \mathrm{~nm}$ thickness. Also, for $6 \mathrm{~nm}$ thickness, superparamagnetic transition sets in at $125 \pm 25 \mathrm{~nm}$ wire width. We therefore show that with $10 \mathrm{~nm}$ thickness, instead of $6 \mathrm{~nm}$ thickness, more miniaturisation of nanowire width can be achieved before the onset of superparamagnetism. This may be seen as very efficient in achieving increased storage density in memory devices.

\section{ACKNOWLEDGEMENTS}

We thank Tertiary Education Trust Fund (TETFund) for sponsoring this work. 


\section{REFERENCES}

1. Jiles, D. C. $2^{\text {nd }}$ ed. London: Chapman \& Hall, (1998)

2. Spaldin, N.A. $2^{\text {nd }}$ ed. Cambridge: Cambridge University Press (2010)

3. Uhlig, W. C., Shi, J. Journal of applied physics. 95, 11 (2004)

4. Allwood, D. A., Xiong, G., Cooke, M. D., Cowburn, R. P. Journal of Physics D: Applied Physics. 36, 18 (2003)

5. Hubert, A. and Schafer, R. Berlin: Springer (1998)

6. Freeman, M .R and Hiebert, W .K. Hillebrands, B. and Ounedjela, K. eds. Berlin: Springer, (2002)

7. Wu, J., Schmool, D. S., Hughes, N. D., Moore, J. R., Hicken, R. J. Journal of applied physics. 91, 1 (2002)

8. Gerrits, T., Van Den Berg, H. A. M., Hohlfeld, J., Bär, L., \& Rasing, T. Nature. 418, 6897 (2002)

9. Cowburn, R. P., Welland, M. E. Science. 287, 5457 (2000)

10. Cowburn, R. P.. Physical Review B. 65, 9 (2002)

11. Allwood, D. A., Xiong, G., Cooke, M. D., Faulkner, C. C., Atkinson, D., Vernier, N., \& Cowburn, R. P. Science. 296, 5575 (2002)

12. Atkinson, D., Allwood, D. A., Xiong, G., Cooke, M. D., Faulkner, C. C., Cowburn, R. P. Nature materials. 2, 2 (2001)

13. Allwood, D. A., Xiong, G., Cooke, M. D., Cowburn, R. P.. Journal of Physics D: Applied Physics. 36, 18 (2003)

14. Allwood, D. A., Xiong, G., Cooke, M. D., Faulkner, C. C., Atkinson, D., Vernier, N., \& Cowburn, R. P. S. Science. 296, 5575 (2002)

15. Atkinson, D., Allwood, D. A., Xiong, G., Cooke, M. D., Faulkner, C. C., Cowburn, R. P. Nature materials. 2, 2 (2003) 